\title{
Evidencia poblacional y análisis crítico de determinación social sobre el uso del condón en Colombia
}

\author{
Population-based evidence and critical analysis of social \\ determinants in the use of condom in Colombia
}

\author{
DraC. Marcela Arrivillaga, MSc. Luis Miguel Tovar, Dr. Diego Correa \\ Pontificia Universidad Javeriana. Cali, Colombia.
}

\section{RESUMEN}

Objetivos: determinar el nivel de uso del condón en población general colombiana y analizar los hallazgos desde el enfoque crítico de la determinación social de la salud. Métodos: estudio transversal realizado con datos de la Encuesta Nacional de Salud, 2007. La muestra estuvo conformada por 29760 registros de personas entre 18 y 69 años de las principales regiones de Colombia.

Resultados: entre el 75 y 94 \% de los encuestados habían oído hablar del condón pero a pesar de eso, su uso arrojó porcentajes por debajo de 22,2, y fueron las mujeres quienes menos lo utilizaron. Las personas con bajos niveles de escolaridad, sin escolaridad, afiliados al régimen subsidiado para población pobre y desplazados, fueron quienes usaron menos el condón, tanto en la primera como en la última relación sexual. De forma complementaria, se encontró que en una submuestra de 209 personas, solo $13(6,5 \%)$ utilizaron el condón como medida preventiva cuando tuvieron alguna infección de transmisión sexual.

Conclusiones: El uso del condón en la población colombiana es bajo, existen determinaciones sociales de tipo estructural relacionadas con este hecho, como son el acceso a la educación y a servicios de salud, así como patrones culturales de vulnerabilidad relacionados con el género. La promoción del uso del condón en el país debe pasar por decisiones de la autoridad sanitaria encabezada por el Ministerio de Salud, con participación de otros sectores involucrados en la calidad de vida de los colombianos.

Palabras clave: uso de condón, población general, determinantes sociales de la salud, Colombia. 


\section{ABSTRACT}

Objectives: to determine the extent of the use of condom by the Colombian general population and to analyze the findings from a critical approach to the social determinants of health.

Methods: a cross-sectional study using data from the National Health Survey of 2007 was conducted. The sample was made up of 29760 records of people aged 18 to 69 years from the main regions of Colombia.

Results: in spite of the fact that 75 to $94 \%$ of the surveyed people had heard about the condom, less than $22.2 \%$ of them used it and the females showed the lowest indexes. Those persons with low schooling, no education at all, included in the subsidy system for the poor, and the displaced people were the ones who used the condom less in their first and their most recent sexual intercourses. Additionally, it was found that $13(6.5 \%)$ out of 209 people in a subsample used condom as a preventive action when they suffered some sexually transmitted infection.

Conclusions: the use of condom by the Colombian population is low; there are structural-type social determinants related to this event like access to education and health services as well as gender-dependent cultural patterns of vulnerability. Promotion of condom nationwide should be supported by health authority decisions led by the Ministry of Public Health, with the participation of other sectors involved in the quality of life of the Colombian citizens.

Key words: use of condom, general population, social determinants of health, Colombia.

\section{NTRODUCCI ÓN}

A nivel mundial, el embarazo adolescente, las infecciones de transmisión sexual (ITS) y el $\mathrm{VIH} /$ sida se encuentran entre los principales problemas de salud pública. La mayoría de las estrategias diseñadas a lo largo del mundo para el control y la prevención de estas enfermedades reconocen que el uso correcto y consistente del condón es una de las principales medidas de protección para reducir las altas tasas de incidencia y prevalencia de estos eventos. ${ }^{1-3}$ En Colombia, por ejemplo, en el Plan Nacional de Respuesta ante el VIH y el sida 2008-2011 se incluyó un presupuesto del $10 \%$ de los recursos ( $\sim 6$ millones de dólares) para el suministro de condones. ${ }^{4}$

Los estudios realizados en este país sobre uso del condón muestran un panorama similar a los hallazgos en otros países de América Latina. ${ }^{2,5}$ Una investigación realizada con adultos muestra que el $30,6 \%$ de la muestra nunca usa condón en penetraciones vaginales, $71 \%$ nunca con sus parejas regulares y el $88 \%$ nunca o pocas veces con parejas ocasionales; además, el $45 \%$ no utiliza este método de protección en relaciones sexuales penetrativas bajo efecto de alcohol o drogas. ${ }^{6}$ Otro estudio con jóvenes encuentra cifras similares, solo el 17,3 \% usa el condón en todas las relaciones sexuales con penetración vaginal, $80,5 \%$ no lo utiliza durante el sexo oral, $54,1 \%$ nunca lo usa en penetración anal y el $66,1 \%$ no lo hizo en su última relación sexual. Una de las principales razones para no usar condón es la percepción de confianza en la persona con quien se tiene la relación sexual. ${ }^{7}$ 
A pesar de estos y otros datos publicados en Colombia, lo cierto es que en el país, a nivel poblacional la información sobre uso del condón es limitada. Los estudios disponibles muestran el comportamiento de grupos específicos como adolescentes y jóvenes, mujeres en edad fértil, población con VIH o vulnerable al sida y trabajadores sexuales. Esto dificulta la orientación y el diseño de políticas y planes que promuevan efectivamente el uso del condón, tanto para estos subgrupos como para la población general.

Dada la escasez de información en esta materia, este estudio tiene como objetivo determinar el nivel de uso del condón en población general colombiana de 18 a 69 años, a partir del análisis de la evidencia poblacional más reciente en el país. Este artículo contextualiza los resultados encontrados desde el enfoque crítico de la determinación social de la salud. ${ }^{8}$

\section{MÉTODOS}

Estudio descriptivo realizado con los datos de la Encuesta Nacional de Salud, realizada en Colombia en 2007 (ENS-2007), con autorización del Ministerio de la Protección Social y aprobación del Comité de Ética de la Pontificia Universidad Javeriana de Cali.

\section{Población y muestra}

Se contó con información contenida en 29760 registros de individuos seleccionados de un total de 96958 elegibles provenientes de 41543 hogares de las zonas urbanas y rurales de Colombia. Para la obtención de los datos, se utilizó un muestreo probabilístico, polietápico, estratificado y por conglomerados.

\section{Instrumentos}

La ENS-2007 estuvo conformada por cuatro encuestas entre ellas una "encuesta de hogares" que constaba de cuatro módulos. Para este estudio, se analizó la información sociodemográfica de los módulos 1 y 2 , junto con la información sobre actividad sexual y conocimiento y uso del condón, del módulo 4.

\section{Análisis de datos}

Con la ayuda de estadísticas descriptivas se realizó una caracterización sociodemográfica de la muestra estudiada. Se estimaron las proporciones poblacionales e intervalos de confianza para aspectos relacionados con el uso del condón. Las estimaciones se hicieron ajustando por el diseño de muestreo y las respectivas ponderaciones. El análisis de datos fue realizado con la ayuda del software SPSS v17 y se estableció un nivel de confianza del $95 \%$ para obtener los resultados.

\section{RESULTADOS}

La muestra correspondió a 29760 personas entre 18 y 69 años de las principales regiones de Colombia. Se destaca que hubo representación significativa de los 
diferentes grupos de edad, un poco inferior en la población mayor de 55 años debido a la estructura demográfica del país. En cuanto a escolaridad, el mayor porcentaje de los encuestados correspondió a básica secundaria y media con el 37,1 \% y el analfabetismo estuvo presente en el $6,1 \%$. En cuanto a zona de residencia, predominó la población residente en zonas urbanas con el 76,6 \%. La información completa sobre los datos sociodemográficos se presentan en la tabla 1.

Tabla 1. Características sociodemográficas en adultos de 18 a 69 años Colombia, $2007(n=29760)$

\begin{tabular}{|c|c|c|c|c|c|}
\hline \multicolumn{2}{|c|}{ Características sociodemográficas } & \multirow{2}{*}{$\frac{n}{12886}$} & \multirow{2}{*}{$\begin{array}{c}\% \\
46,3\end{array}$} & \multicolumn{2}{|c|}{ IC: $95 \%$} \\
\hline \multirow{2}{*}{ Sexo } & Hombre & & & 45,6 & 47,0 \\
\hline & Mujer & 16874 & 53,7 & 53,0 & 54,4 \\
\hline \multirow{5}{*}{ Grupo de edad (años) } & 18 a 24 & 5163 & 20,2 & 19,7 & 20,8 \\
\hline & 25 a 34 & 7000 & 23,8 & 23,2 & 24,4 \\
\hline & 35 a 44 & 6389 & 21,7 & 21,2 & 22,1 \\
\hline & 45 a 54 & 5776 & 18,1 & 17,6 & 18,6 \\
\hline & 55 a 69 & 5432 & 16,2 & 15,7 & 16,8 \\
\hline \multirow{7}{*}{ Nivel de escolaridad } & Ninguno & 2156 & 6,1 & 5,3 & 7,0 \\
\hline & Preescolar & 42 & 0,1 & 0,1 & 0,2 \\
\hline & Básica primaria & 10171 & 31,6 & 29,8 & 33,5 \\
\hline & Básica secundaria y media & 10750 & 37,1 & 35,8 & 38,5 \\
\hline & Técnico a tecnológico & 1793 & 6,8 & 5,9 & 7,8 \\
\hline & Universidad & 2233 & 8,5 & 8,0 & 9,1 \\
\hline & Sin dato & 2615 & 9,7 & 9,2 & 10,2 \\
\hline \multirow{7}{*}{$\begin{array}{l}\text { Régimen de Seguridad } \\
\text { Social en Salud }\end{array}$} & Contributivo & 9484 & 38,3 & 33,6 & 43,2 \\
\hline & Subsidiado & 12603 & 36,7 & 33,3 & 40,3 \\
\hline & Especiales & 1223 & 3,6 & 3,2 & 4,1 \\
\hline & Otra Entidad & 885 & 2,1 & 1,3 & 3,2 \\
\hline & Carta desplazados & 97 & 0,3 & 0,2 & 0,4 \\
\hline & Ninguna & 5298 & 18,3 & 16,6 & 20,2 \\
\hline & No sabe & 170 & 0,7 & 0,5 & 0,8 \\
\hline \multirow{2}{*}{ Zona de residencia } & Cabecera & 22761 & 76,6 & 71,4 & 81,0 \\
\hline & Resto & 6999 & 23,4 & 19,0 & 28,6 \\
\hline
\end{tabular}

En la muestra de estudio se encontró que entre el 75 y el $94 \%$ de las personas encuestadas, independientemente del género o del tipo de afiliación al sistema de Seguridad Social en Salud, habían oído hablar del condón. No obstante, el conocimiento sobre este método de protección es estadísticamente diferencial por grupos de edad: a medida que aumentó la edad, el conocimiento era menor $(p=0,000)$. También se observaron cambios estadísticamente significativos según escolaridad, con menor conocimiento del condón en personas sin escolaridad o solo con estudios de primaria $(p=0,000)$ (tabla 2$)$.

Solo el $16,4 \%$ de los encuestados manifestaron que usaron condón en su primera relación sexual, sin diferencias estadísticas importantes entre hombres y mujeres. Los más jóvenes $(p=0,000)$ fueron quienes más informaron usar el método de protección en esta relación. Al analizar los datos según escolaridad, se encontró menor 
utilización de este método de protección en personas con niveles bajos de escolaridad o sin escolaridad $(p=0,000)$, entre los afiliados al régimen subsidiado de salud (para población pobre) y en población con carta de desplazados $(p=0,016)$, como aparece en la tabla 3.

Con respecto al uso del condón en la última relación sexual, el 17,4 \% lo utilizó; en particular, los hombres $(22,1 \%)$, y las mujeres $(13,7 \%)$, con una diferencia estadísticamente significativa $(p=0,000)$. De manera consistente con la utilización del condón en la primera relación sexual, los jóvenes notificaron un mayor uso, hasta el $35,3 \%$ en el rango de edad de 18 a 24 años. Sobresale que el $80 \%$ de las personas mayores a 45 años no usaron el condón en la última relación sexual. Respecto a otras variables, sigue siendo menor el uso del condón en población con baja o sin escolaridad $(p=0,000)$, personas del régimen subsidiado o con carta de desplazados $(p=0,004)$, como se aprecia en la tabla 4 .

Por último, al estudiar las prácticas de prevención de transmisión de ITS, como complemento a los datos ya obtenidos, se les preguntó a las de 209 personas que conformaron una submuestra: Cuando usted tuvo la última ITS, ¿hizo alguna de las siguientes cosas para evitar infectar a la(s) persona(s) con quien(es) tenía relaciones sexuales? Se encontró que solo $13(6,5 \%)$ personas, usaron el condón como medida.

Las prácticas más utilizadas para el manejo de las ITS fueron tomar medicamentos $93,3 \%$, dejar de tener relaciones sexuales $78,4 \%$; otras pericias fueron, asearse $20,6 \%$, eliminar ladillas, piojos y similares $16,3 \%$, y rasurarse los genitales $7,2 \%$.

Tabla 2. Porcentaje de personas 18 a 69 años con registro de actividad sexual y conocimiento del condón según características sociodemográficas. Colombia. 2007

\begin{tabular}{|c|c|c|c|c|c|c|c|c|c|}
\hline \multirow{3}{*}{$\begin{array}{l}\text { Características } \\
\text { sociodemográficas }\end{array}$} & \multicolumn{6}{|c|}{ ¿Ha oído hablar del condón? } & \multirow{2}{*}{\multicolumn{2}{|c|}{ Total }} & \multirow{3}{*}{$p$} \\
\hline & \multicolumn{3}{|c|}{ Sí } & \multicolumn{3}{|c|}{ No } & & & \\
\hline & $\%$ & \multicolumn{2}{|c|}{ IC: $95 \%$} & $\%$ & \multicolumn{2}{|c|}{ IC: $95 \%$} & $\%$ & $n$ & \\
\hline \multicolumn{10}{|l|}{ Sexo } \\
\hline Hombre & 92,2 & 90,6 & 93,6 & 7,8 & 6,4 & 9,4 & 100,0 & 12033 & 0,294 \\
\hline Mujer & 92,0 & 90,4 & 93,3 & 8,0 & 6,7 & 9,6 & 100,0 & 15573 & - \\
\hline \multicolumn{10}{|l|}{ Edad (años) } \\
\hline 18 a 24 & 94,8 & 93,2 & 96,1 & 5,2 & 3,9 & 6,8 & 100,0 & 4469 & 0,000 \\
\hline 25 a 34 & 94,2 & 92,1 & 95,7 & 5,8 & 4,3 & 7,9 & 100,0 & 6688 & - \\
\hline 35 a 44 & 92,8 & 91,2 & 94,1 & 7,2 & 5,9 & 8,8 & 100,0 & 6090 & - \\
\hline 45 a 54 & 90,8 & 89,1 & 92,2 & 9,2 & 7,8 & 10,9 & 100,0 & 5442 & - \\
\hline 55 a 69 & 86,2 & 84,1 & 88,0 & 13,8 & 12,0 & 15,9 & 100,0 & 5008 & - \\
\hline \multicolumn{10}{|l|}{ Escolaridad } \\
\hline Ninguna & 79,1 & 75,7 & 82,1 & 20,9 & 17,9 & 24,3 & 100,0 & 1926 & 0,000 \\
\hline Preescolar & 98,1 & 88,1 & 99,7 & 1,9 & 0,3 & 11,9 & 100,0 & 40 & - \\
\hline Básica primaria & 89,1 & 87,5 & 90,5 & 10,9 & 9,5 & 12,5 & 100,0 & 9539 & - \\
\hline $\begin{array}{l}\text { Básica secundaria } \\
\text { y media }\end{array}$ & 94,6 & 92,6 & 96,1 & 5,4 & 3,9 & 7,4 & 100,0 & 10169 & - \\
\hline Técnico o tecnológico & 96,2 & 94,4 & 97,4 & 3,8 & 2,6 & 5,6 & 100,0 & 1674 & - \\
\hline Universidad & 93,6 & 88,5 & 96,6 & 6,4 & 3,4 & 11,5 & 100,0 & 2110 & - \\
\hline \multicolumn{10}{|l|}{ Régimen de salud } \\
\hline Contributivo & 93,0 & 89,9 & 95,3 & 7,0 & 4,7 & 10,1 & 100,0 & 8867 & 0,052 \\
\hline Subsidiado & 90,5 & 89,1 & 91,8 & 9,5 & 8,2 & 10,9 & 100,0 & 11687 & - \\
\hline Especiales & 93,9 & 91,5 & 95,6 & 6,1 & 4,4 & 8,5 & 100,0 & 1159 & - \\
\hline Otra entidad & 86,8 & 82,5 & 90,1 & 13,2 & 9,9 & 17,5 & 100,0 & 800 & - \\
\hline Carta desplazados & 96,3 & 80,3 & 99,4 & 3,7 & 0,6 & 19,7 & 100,0 & 92 & - \\
\hline Ninguna & 93,2 & 91,6 & 94,5 & 6,8 & 5,5 & 8,4 & 100,0 & 4944 & - \\
\hline
\end{tabular}

http://scielo.sld.cu 
Revista Cubana de Salud Pública 2012; 38(4): 553-561

Tabla 3. Uso del condón en personas de 18 a 69 años en primera relación sexual. Colombia, 2007

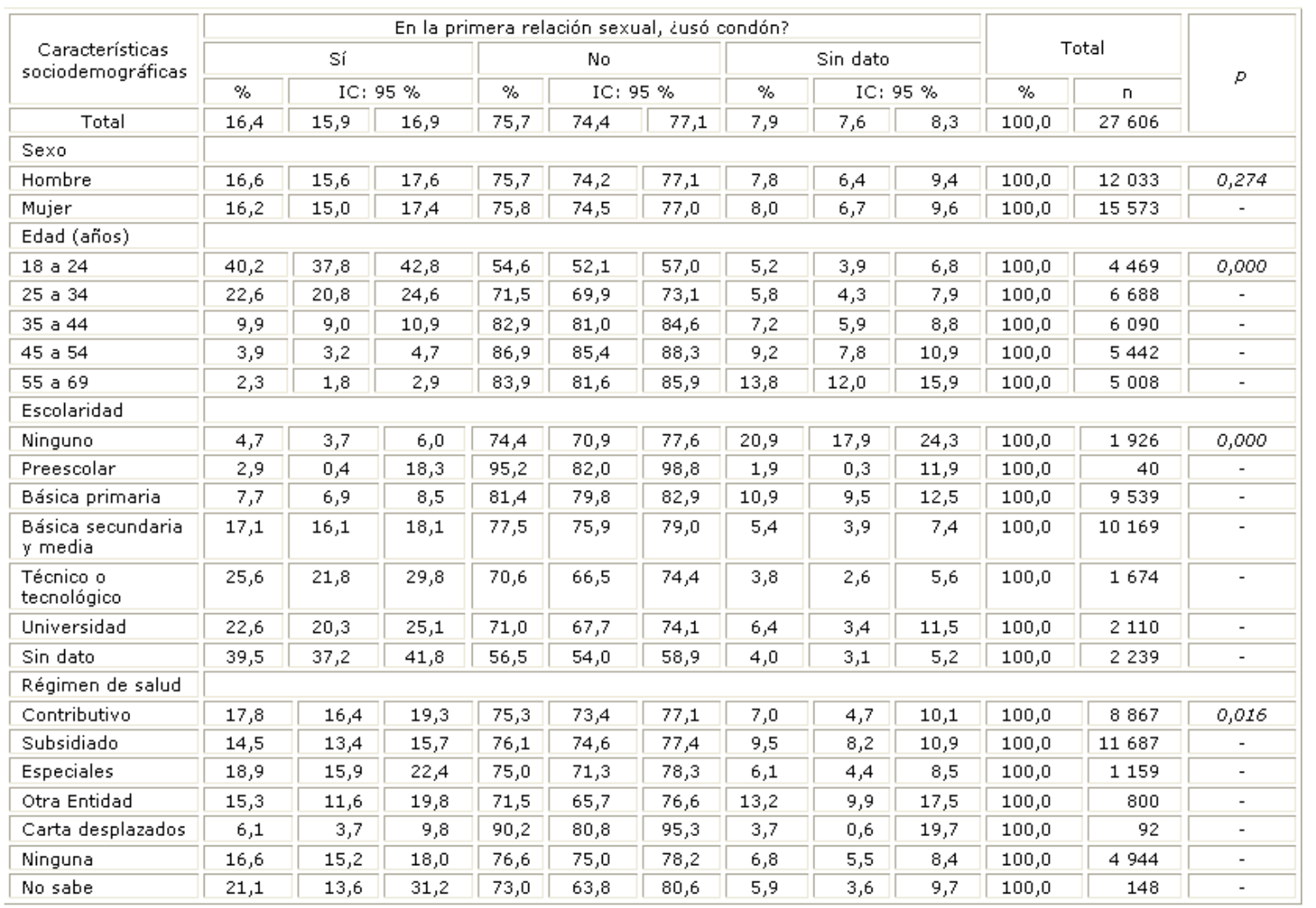

Tabla 4. Uso del condón en personas de 18 a 69 años en última relación sexual. Colombia, 2007

\begin{tabular}{|c|c|c|c|c|c|c|c|c|c|c|c|c|}
\hline \multirow{4}{*}{$\begin{array}{l}\text { Características } \\
\text { sociodemográficas }\end{array}$} & \multicolumn{9}{|c|}{ La última vez que tuvo relaciones sexuales, ¿usted o su pareja usó condón? } & \multirow{2}{*}{\multicolumn{2}{|c|}{ Total }} & \multirow{4}{*}{$p$} \\
\hline & \multicolumn{3}{|c|}{ Sí } & \multicolumn{3}{|c|}{ No } & \multicolumn{3}{|c|}{ Sin dato } & & & \\
\hline & \multirow{2}{*}{$\begin{array}{c}\% \\
17,4\end{array}$} & \multicolumn{2}{|c|}{$\mathrm{IC}=95 \%$} & \multirow{2}{*}{$\begin{array}{c}\% \\
74,7\end{array}$} & \multicolumn{2}{|c|}{$\mathrm{IC}=95 \%$} & \multirow{2}{*}{$\frac{\%}{7,9}$} & \multicolumn{2}{|c|}{$\mathrm{IC}=95 \%$} & \multirow{2}{*}{$\begin{array}{c}\% \% \\
100,0 \\
\end{array}$} & \multirow{2}{*}{$\frac{n}{27606}$} & \\
\hline & & 16,8 & 17,9 & & 73,4 & 76,1 & & 7,6 & 8,3 & & & \\
\hline \multicolumn{13}{|l|}{ Sexo } \\
\hline Hombre & 22,1 & 21,2 & 23,0 & 70,1 & 68,6 & 71,6 & 7,8 & 6,4 & 9,4 & 100,0 & 12033 & 0,000 \\
\hline Mujer & 13,7 & 13,0 & 14,5 & 78,2 & 76,4 & 79,9 & 8,0 & 6,7 & 9,6 & 100,0 & 15573 & - \\
\hline \multicolumn{13}{|l|}{ Edad (años) } \\
\hline 18 a 24 & 35,3 & 33,4 & 37,2 & 59,5 & 57,1 & 61,9 & 5,2 & 3,9 & 6,8 & 100,0 & 4469 & 0,000 \\
\hline 25 a 34 & 20,5 & 19,3 & 21,7 & 73,7 & 71,7 & 75,6 & 5,8 & 4,3 & 7,9 & 100,0 & 6688 & - \\
\hline 35 a 44 & 14,6 & 13,7 & 15,6 & 78,2 & 76,6 & 79,7 & 7,2 & 5,9 & 8,8 & 100,0 & 6090 & - \\
\hline 45 a 54 & 10,4 & 9,3 & 11,5 & 80,4 & 78,7 & 82,1 & 9,2 & 7,8 & 10,9 & 100,0 & 5442 & - \\
\hline 55 a 69 & 5,5 & 4,7 & 6,4 & 80,7 & 78,6 & 82,6 & 13,8 & 12,0 & 15,9 & 100,0 & 5008 & - \\
\hline \multicolumn{13}{|l|}{ Escolaridad } \\
\hline Ninguno & 8,7 & 7,5 & 10,2 & 70,4 & 66,8 & 73,7 & 20,9 & 17,9 & 24,3 & 100,0 & 1926 & 0,000 \\
\hline Preescolar & 13,3 & 4,8 & 31,9 & 84,8 & 64,8 & 94,4 & 1,9 & 0,3 & 11,9 & 100,0 & 40 & - \\
\hline Básica primaria & 10,1 & 9,2 & 11,0 & 79,0 & 77,6 & 80,4 & 10,9 & 9,5 & 12,5 & 100,0 & 9539 & - \\
\hline $\begin{array}{l}\text { Básica secundaria } \\
\text { y media }\end{array}$ & 18,3 & 17,2 & 19,5 & 76,3 & 74,0 & 78,4 & 5,4 & 3,9 & 7,4 & 100,0 & 10169 & - \\
\hline Técnico o tecnológico & 23,1 & 20,9 & 25,5 & 73,1 & 70,2 & 75,8 & 3,8 & 2,6 & 5,6 & 100,0 & 1674 & - \\
\hline Universidad & 23,6 & 21,7 & 25,6 & 70,0 & 66,5 & 73,4 & 6,4 & 3,4 & 11,5 & 100,0 & 2110 & - \\
\hline \multicolumn{13}{|l|}{ Régimen de salud } \\
\hline Contributivo & 17,3 & 16,4 & 18,3 & 75,7 & 73,1 & 78,2 & 7,0 & 4,7 & 10,1 & 100,0 & 8867 & 0,004 \\
\hline Subsidiado & 16,0 & 15,1 & 17,0 & 74,5 & 73,1 & 75,9 & 9,5 & 8,2 & 10,9 & 100,0 & 11687 & - \\
\hline Especiales & 15,8 & 13,7 & 18,1 & 78,1 & 75,3 & 80,7 & 6,1 & 4,4 & 8,5 & 100,0 & 1159 & - \\
\hline Otra Entidad & 18,1 & 14,3 & 22,5 & 68,7 & 63,8 & 73,3 & 13,2 & 9,9 & 17,5 & 100,0 & 800 & \\
\hline Carta desplazados & 6,5 & 2,9 & 13,9 & 89,9 & 78,7 & 95,5 & 3,7 & 0,6 & 19,7 & 100,0 & 92 & - \\
\hline Ninguna & 21,7 & 20,1 & 23,3 & 71,5 & 69,3 & 73,5 & 6,8 & 5,5 & 8,4 & 100,0 & 4944 & - \\
\hline No sabe & 23,2 & 17,5 & 30,1 & 70,8 & 63,8 & 77,0 & 5,9 & 3,6 & 9,7 & 100,0 & 148 & - \\
\hline
\end{tabular}




\section{DISCUSIÓN}

A partir del análisis de una muestra representativa a nivel de país, este estudio reveló que colombianos entre 18 y 69 años tienen una baja utilización del condón, como medio de protección en las relaciones sexuales. A pesar que entre el 75 y el $94 \%$ de los encuestados a nivel nacional ha oído hablar del condón, su uso mostró niveles por debajo de $22,2 \%$, siendo las mujeres quienes menos lo utilizaron. Se encontró además que, tanto en la primera como en la última relación sexual, personas con bajos niveles de escolaridad, sin escolaridad, afiliados al régimen subsidiado y desplazados, utilizaron menos el condón en sus relaciones sexuales. De forma complementaria, resaltó el hallazgo que indica que solo13 $(6,5 \%)$ de los encuestados utilizó el condón como medida preventiva cuanto tuvo alguna ITS.

Con este estudio se corrobora que el solo conocimiento de una medida de protección no implica necesariamente la transferencia de tales conocimientos al nivel de los comportamientos preventivos en salud. En segundo término, queda demostrado que en Colombia existen determinaciones sociales de tipo estructural relacionadas con el uso del condón, como son el acceso a la educación y a servicios de salud, y patrones culturales de vulnerabilidad relacionados con el género. Al respecto, la Política Nacional de Salud Sexual Reproductiva publicada en 2003, cuatro años antes de la recolección de datos de la ENS, ya señalaba entonces que entre las vulnerabilidades más frecuentemente asociadas a la SSR de los colombianos estaban la baja escolaridad, la situación de desplazamiento y las limitaciones de la cobertura del sistema general de seguridad social en salud. Una de las estrategias de la política fue promover el condón a pesar, entre otros, de las barreras culturales para su uso por parte tanto de hombres como de mujeres. No obstante, no solo en el campo de la prevención de ITS y VIH, sino también en lo que respecta al embarazo adolescente, nueve años después de la expedición de la política, los resultados, en especial para las mujeres, no son favorables. De igual forma, los resultados de este estudio cuestionan los efectos del Plan Nacional de Respuesta ante el VIH y el sida 20082011, al menos en cuanto a uso del condón se refiere. La realidad es que en el país hay un incremento gradual en el número de mujeres infectadas por $\mathrm{VIH}^{9,10}$ y que el $19,5 \%$ de las adolescentes está embarazada o ha sido madre. ${ }^{11}$

Resultados similares al presentado en este estudio, con datos regionales en mujeres colombianas, encuentran también un bajo nivel de uso del condón, solo en el $15 \%$ de aquellas con vida sexual activa. ${ }^{12}$ Los aspectos que predominan en las mujeres como argumentos para no exigir el condón tienen que ver con la confianza, percibir estabilidad en la relación afectiva, apariencia de bajo riesgo de la pareja y sumisión. ${ }^{2}$ No obstante, si se profundiza en las determinaciones de género en el país, es urgente expandir el acceso a medios educativos creativos y no solo informativos, avanzar en la ruptura de barreras económicas y culturales que ubican a las mujeres en situación de vulnerabilidad en el ámbito privado y de negociación de la relaciones sexuales, ${ }^{6}$ y reorientar la pedagogía de la educación sexual y su implementación en todo el territorio nacional.

En consonancia con estudios previos que han mostrado asociaciones entre las prevalencias de ITS, ${ }^{13,14}$, VIH y determinantes sociales estructurales como la infraestructura sanitaria, la calidad de las viviendas, la educación, el acceso a los servicios de atención en salud, ${ }^{15}$ este estudio señala que el uso del condón, más allá de estudiarse como un asunto individual, está relacionado con tales determinaciones. Personas en situación de pobreza vinculadas al régimen subsidiado y población en situación de desplazamiento por efecto del conflicto interno que sufre el país, se enfrentan con la debilidad de los planes de salud pública a nivel nacional y territorial, limitada atención en salud sexual y reproductiva, lo que incluye escasa provisión de condones por parte de las entidades promotoras de salud, e insuficientes programas 
de promoción y prevención, con pocos espacios para la promoción de prácticas sexuales seguras.

El impulso al uso del condón en el país debe pasar por decisiones de autoridad sanitaria encabezada por el Ministerio de Salud, con participación de otros sectores involucrados en la calidad de vida de los colombianos. La aplicación de políticas, planes y programas en salud sexual y reproductiva, requiere ajustes, no solo para la intervención efectiva en términos individuales, sino también la adecuada inclusión de determinantes sociales estructurales, transversalizados por la economía y la cultura.

Finalmente, es preciso señalar que los resultados del análisis aquí presentado pueden contener sesgos derivados de la naturaleza misma de la ENS-2007. El carácter transversal de esta encuesta y la recolección de información privada en el contexto de los hogares marcan limitaciones al estudio, que deben ser subsanadas en investigaciones siguientes. Sin embargo, los resultados que se han mostrado pueden ser de utilidad para orientar la toma de decisiones tanto en el nivel político como en ámbitos académicos interesados en el tema de la salud sexual reproductiva en Colombia.

\section{Agradecimientos}

Los autores agradecen al Ministerio de la Protección Social por la autorización concedida para realizar análisis relacionados con la salud sexual y reproductiva de los colombianos, entre los cuales se encuentra el estudio aquí presentado, a la Pontificia Universidad Javeriana Cali, por la ayuda prestada. De igual forma, reconocen la contribución de Helmer Zapata en el análisis de los datos.

\section{REFERENCI AS BI BLI OGRÁFICAS}

1. Caballero A, Toro I, Sánchez F, Carrera P. Las emociones y el género en la conducta sexual de riesgo en adolescentes. Revista Psicología Salud. 2009;24(3):34961.

2. Caballero A, Villaseñor A. El estrato socioeconómico como factor predictor del uso constante de condón en adolescentes. Rev Saúde Pública. 2001;35(6):531-8.

3. Icunacurí M. Comunicación sexual en adolescentes y su implicación en la consistencia del uso del condón. Enseñanza Investigación Psicología. 2010;15(1):10729.

4. Ministerio de la Protección Social. Plan nacional de respuesta ante el VIH y el sida. Colombia 2008-2011. Bogotá: Ministerio; 2008.

5. Soto V. Factores asociados al no uso del condón. Estudio en adolescentes y adultos jóvenes en Chiclayo. An Fac Med Lima. 2006;67(2):152-9.

6. González J M, Herrera D, Ruiz M. Comportamientos y conocimientos sexuales relacionados con VIH/sida en postgrado. Psicogente. 2006; 9(15): 146-56.

7. Contreras F, Carrobles JA, Juárez-Acosta F. Propiedades psicométricas del "Cuestionario confidencial de vida sexual activa" para evaluar conductas de riesgo en VIH-sida. Psicología Conductual. 2007; 15(1):5-27. 
8. Breilh J. Epidemiología crítica. Ciencia emancipadora e interculturalidad. Buenos Aires: Lugar Editorial; 2003.

9. Ministerio de la Protección Social. Resumen de situación de la epidemia por VIH/sida en Colombia 1983 a 2008. Bogotá: Ministerio, Oficina Asesora de Comunicaciones; 2009.

10. UNAIDS. Report on the global AIDS epidemic. Geneve: UNAIDS; 2010.

11. Profamilia. Encuesta Nacional de Demografía y Salud Bogotá: ENDS; 2010.

12. Amado MA, Vega BR, Jiménez, MC, Piña JA. Factores que influyen en el uso del preservativo en mujeres en edad reproductiva de Tunja, Colombia. Acta Colombiana Psicol. 2007; 10(2): 143-51.

13. Arrivillaga M, Zapata H, Tovar LM, Correa D, Varela MT, Hoyos PA. Infecciones de transmisión sexual en Colombia: Análisis basado en la Encuesta Nacional de Salud Año 2007. Rev Gerencia Políticas Salud. 2011; 10(20):69-80.

14. Arrivillaga M, Correa D, Tovar LM, Zapata H, Varela MT, Hoyos PA. Infecciones de transmisión sexual en la región pacífica colombiana: implicaciones para población en situación de vulnerabilidad étnica, social y económica. Pensamiento Psicológico. 2011; 9(16): 145-52.

15. Tovar LM, Arrivillaga M. VIH/sida y determinantes sociales estructurales en municipios del Valle del Cauca-Colombia. Rev Gerencia Políticas Salud. 2011;21(10): 112-23.

Recibido: 22 de febrero de 2012.

Aprobado: 26 de marzo de 2012.

Marcela Arrivillaga Quintero. Departamento de Salud Pública y Epidemiología. Grupo Salud y Calidad de Vida. Pontificia Universidad Javeriana de Cali. Cali, Colombia.

Teléf.: (572) 4856400 Ext. 8681. Correo electrónico: marceq@javerianacali.edu.co 